\title{
Safety and Efficacy of Vedolizumab in a Patient With Crohn's Disease After Developing An Active Tuberculosis Infection Associated With Infliximab- Biosimilar: A Case Report
}

\section{Yuya Sugiyama}

Asahikawa Medical University: Asahikawa lka Daigaku https://orcid.org/0000-0001-5376-4256

Nobuhiro Ueno ( $D$ u-eno@asahikawa-med.ac.jp)

Asahikawa Medical University: Asahikawa Ika Daigaku https://orcid.org/0000-0001-5448-4766

Shion Tachibana

Asahikawa Medical University: Asahikawa Ika Daigaku

Yu Kobayashi

Asahikawa Medical University: Asahikawa Ika Daigaku

Yuki Murakami

Asahikawa Medical University: Asahikawa Ika Daigaku

Takahiro Sasaki

Asahikawa Medical College: Asahikawa Ika Daigaku

Takehito Kunogi

Asahikawa Medical College: Asahikawa Ika Daigaku

Keitaro Takahashi

Asahikawa Medical University: Asahikawa Ika Daigaku

Katsuyoshi Ando

Asahikawa Medical University: Asahikawa Ika Daigaku

Shin Kashima

Asahikawa Medical University: Asahikawa Ika Daigaku

Kentaro Moriichi

Asahikawa Medical University: Asahikawa Ika Daigaku Hiroki Tanabe

Asahikawa Medical University: Asahikawa Ika Daigaku

Toshikatsu Okumura

Asahikawa Medical University: Asahikawa Ika Daigaku Mikihiro Fujiya

Asahikawa Medical University: Asahikawa Ika Daigaku 


\section{Case report}

Keywords: Mycobacterium tuberculosis, Infliximab, Vedolizumab, Crohn's disease, active tuberculosis, latent tuberculosis

Posted Date: May 26th, 2021

DOl: https://doi.org/10.21203/rs.3.rs-552372/v1

License: (c) (i) This work is licensed under a Creative Commons Attribution 4.0 International License.

Read Full License 


\section{Abstract}

Background: Screening of latent tuberculosis (TB) before the induction of anti-tumor necrosis factor (antiTNF) agents is very important to prevent the reactivation of TB. However, active TB can still occur, and the ideal therapeutic strategy for IBD patients who develop active TB has not been established.

Case presentation: A 21-year-old Vietnamese man with active ileo-colonic Crohn's disease visited our hospital. While computed tomography (CT) revealed a nodular lesion at the right lung, a serological interferon gamma release assay (IGRA) and several culture tests were all negative. The intravenous administration of infliximab-biosimilar (IFX-BS) with an immunomodulator were initiated. After the induction therapy, he achieved clinical remission. However, he presented with a high fever 17 weeks after the initiation. CT revealed the expansion of the nodular lesion at the right lung, and serological IGRA was positive. He was diagnosed with reactivation of latent TB. He had not achieved mucosal healing when immunosuppressive therapy was discontinued. Thus, he was administered vedolizumab (VDZ), which was considered not to influence the TB status, as maintenance therapy. Consequently, he achieved mucosal healing without relapse of active TB.

Conclusions: This is the first report to describe the safe usage of VDZ as maintenance therapy without the induction of TB relapse in a CD patient. In Asian countries, clinicians must be alert for the reactivation of latent TB during the administration of anti-TNF agents. VDZ might be a safe option for maintenance therapy in CD patients, even in cases with active TB.

\section{Background}

Anti-tumor necrosis factor (anti-TNF) agents have a high therapeutic efficacy and safety profile for inflammatory bowel disease (IBD), but a small percentage of patients experience severe adverse effects, including infectious disease. ${ }^{1}$ TNF-alpha plays an important role in the host defense against Mycobacterium tuberculosis, so anti-TNF agents are associated with an increased risk of active tuberculosis (TB) ${ }^{2}$

Most cases of active TB develop within several months after the initiation of anti-TNF agents, suggesting that they are caused by the reactivation of latent TB. ${ }^{3}$ Screening of latent TB before the induction of antiTNF agents is important for preventing the reactivation of TB. Different scientific organizations from all over the world have published guidelines and recommendations concerning active and latent TB. ${ }^{4-6}$ However, active TB can still occur in IBD patients treated with anti-TNF agents despite compliance with recommended preventive measures. ${ }^{7}$ Furthermore, no therapeutic strategy for IBD patients who develop active TB associated with anti-TNF agents has yet been established.

Vedolizumab (VDZ), a gut-selective antibody to a4 47 integrin for the treatment of IBD, has a favorable safety profile with low incidence rates of serious infections, infusion-related reactions and malignancies. ${ }^{8}$ However, its safety for patients who develop active TB associated with anti-TNF agents remains unclear. 
We herein report the successful continuation of VDZ as maintenance therapy without the induction of TB relapse in a CD patient who developed active TB associated with infliximab-biosimilar (IFX-BS).

\section{Case Presentation}

A 21-year-old Vietnamese man presented to Asahikawa Medical University Hospital with hemorrhagic stool. He did not have any personal or family history of IBD or TB. A physical examination revealed the following: body height, $171.0 \mathrm{~cm}$; body weight, $63.8 \mathrm{~kg}$; body mass index, 21.8 ; body temperature, $36.7^{\circ} \mathrm{C}$; blood pressure, $115 / 58 \mathrm{mmHg}$; heart rate, 71 beats $/ \mathrm{min}$; and $\mathrm{SpO}_{2}, 99 \%$ on room air. A laboratory examination revealed high levels of inflammation markers, mild anemia and hypoalbuminemia: white

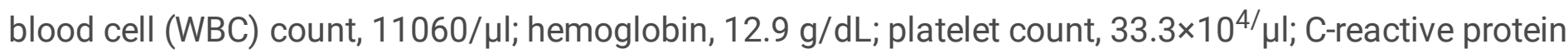
(CRP), $4.43 \mathrm{mg} / \mathrm{dL}$; and albumin, $3.7 \mathrm{~g} / \mathrm{dL}$. Serological tests for infection markers, including the interferon gamma release assay (IGRA), were all negative.

Upper gastrointestinal endoscopy showed multiple vertical small erosions in the duodenum. Small bowel capsule endoscopy (SBCE) showed multiple small ulcers in the jejunum and multiple longitudinal ulcers in the ileum. Colonoscopy showed multiple longitudinal ulcers and a cobblestone appearance in the terminal ileum and multiple small erosions and aphtha throughout the colon (Fig. 1A). An intestinal juice culture examination and polymerase chain reaction (PCR) for any bacteria, including acid-fast bacilli, showed negative findings. Contrast-enhanced computed tomography (CT) revealed a nodular lesion at the apex of the right lung and thickening of the wall with a contrast effect at the terminal ileum (Fig. 2A). A pathological examination did not demonstrate granuloma at the longitudinal ulcer in the terminal ileum.

He was diagnosed with ileo-colonic $C D$ based on the endoscopic findings. The nodular lesion at the apex of the right lung was suspected of being latent TB. However, his serological IGRA and several culture tests (including sputum, urine and intestinal juice) were all negative. Thus, he did not receive a diagnosis of latent TB. His disease activity was a Crohn's disease activity index (CDAl) of 167, Lewis score of 1368 and simple endoscopic score for Crohn's disease (SES-CD) of 13. Because of his young age and the presence of high-activity inflammatory lesion throughout the small intestine and colon, the intravenous administration of IFX-BS with an immunomodulator was initiated as induction therapy. His CDAI and CRP levels immediately decreased after the induction of IFX-BS.

However, at 17 weeks after the initiation of IFX-BS, he visited our hospital with a high fever. A laboratory examination revealed high levels of inflammation markers: WBC count, 6560/ $\mathrm{ll}$; and CRP, $10.33 \mathrm{mg} / \mathrm{dL}$. CT revealed the expansion of the nodular lesion at the apex of the right lung (Fig. 2B). Furthermore, his serological IGRA and acid-fast bacillus culture tests of gastric juice had all turned positive. He was diagnosed with reactivation of latent TB. IFX-BS with an immunomodulator was immediately discontinued, and anti-TB therapy with polypharmacy of rifampicin, isoniazid, ethambutol and pyrazinamide was initiated. At 10 weeks after the initiation of anti-TB therapy, his general condition and inflammation markers had recovered: WBC count, 4850/ul; CRP, $<0.10 \mathrm{mg} / \mathrm{dL}$. 
In July 2019, he had maintained clinical remission of CD without medication. However, colonoscopy still showed an active ulcer in the terminal ileum (Fig. 1B). Regarding his disease activity, he had a CDAI of 85 and SES-CD of 4. His endoscopic findings were improved compared with before induction therapy of IFX$\mathrm{BS}$, but he had not achieved mucosal healing. Thus, another intervention therapy for CD that would not induce a relapse of active TB was considered. VDZ has a favorable safety profile with low incidence rates of serious infections, so VDZ was selected for maintenance therapy.

In June 2020, all of his ulcerative lesions in the small intestine and colon had improved and changed to ulcer scars on SBCE and colonoscopy (Fig. 1C). Regarding his disease activity, he had a CDAI of 80, Lewis score of 0 and SES-CD of 0 . CT revealed shrinkage of the nodular lesion at the apex of the right lung (Fig. 2C). Thus, he achieved both clinical remission and mucosal healing by maintenance therapy with VDZ without relapse of active TB.

\section{Discussion And Conclusions}

This is the first case report to describe how VDZ was able to be continued as maintenance therapy without relapse of TB in a CD patient who developed active TB associated with IFX-BS. VDZ might be a new therapeutic strategy for CD patients who develop active TB.

TNF-alpha plays a central role in the host immune defense against TB infection by inducing the formation and maintenance of granulomas, which prevent the dissemination of infection. Therefore, inhibiting TNF-alpha may increase susceptibility to TB infection and accelerate the reactivation of latent TB infection. ${ }^{2}$ The prevalence of latent TB infection is much higher in Asian countries than in Western countries. The Asian Organization for Crohn's and Colitis (AOCC) recommends that IBD patients be screened for TB by chest radiography and a tuberculin skin test (TST) and/or IGRA before the initiation of anti-TNF agents. ${ }^{5,6}$

In the present case, CT revealed a nodular lesion at the apex of the right lung. However, he did not have any symptoms, and his IGRA and several culture tests as well as a PCR examination were all negative. Therefore, he was not diagnosed with latent TB infection. Lu et al. reported that IGRA showed a superior capability to the TST for diagnosing TB because of its markedly greater sensitivity and specificity. ${ }^{9}$ However, Santos et al. reported that host factors, including inflammatory disease and pulmonary TB, are associated with false-negative IGRA results. ${ }^{10}$ Thus, the ideal way to diagnose latent TB has not yet been established. Even if IGRA is negative, as in our case, IBD patients with pulmonary lesions on CT should receive chemoprophylaxis before the initiation of anti-TNF agents.

The AOCC suggests that anti-TNF agents be withheld when active TB is diagnosed during the administration of anti-TNF agents. ${ }^{6}$ However, the British guideline contrarily recommend that anti-TNF agents be continued if clinically indicated. ${ }^{4}$ Thus, there is little consensus on the impact of immunosuppressive therapy, including anti-TNF agents, on the risk of active TB. In the present case, active lesion in the small intestine remained when the anti-TNF agent with an immunomodulator was 
discontinued. Achieving mucosal healing in CD patients is associated with improved rates of long-term clinical remission; accordingly, such healing is considered to be a crucial therapeutic target. ${ }^{11}$

VDZ is a humanized monoclonal antibody that selectively binds to the $a_{4} \beta_{7}$ integrin. VDZ inhibits the adhesion of lymphocytes to mucosal addressin cell adhesion molecule-1 (MAdCAM-1) and subsequent

migration. Several clinical trials have demonstrated that VDZ has long-term safety and efficacy. ${ }^{8,12}$ According to the results of the post-marketing surveillance of VDZ, only 3 out of approximately 30,000 patients had reactivation of latent TB. ${ }^{13}$ Furthermore, in a retrospective study of IBD patients who received biologic therapy with latent $T B$ infection, there were no cases of reactivation of TB in any of the 10 patients who were treated with VDZ. ${ }^{14}$ Thus, we suspect that VDZ might have a safe profile as maintenance therapy even in patients who develop active TB based on these previous reports describing the extremely low risk of reactivation of latent TB. The present patient has now achieved and maintained complete mucosal healing without relapse of active TB.

In conclusion, this is the first case report describing a patient in whom VDZ was able to be continued as maintenance therapy without inducing relapse of TB in a CD patient who developed active TB associated with IFX-BS. In Asian countries, clinicians must be alert for the reactivation of latent TB during the administration of anti-TNF agents. VDZ might be a safe option for induction and maintenance therapy in CD patients, even in cases with active TB. A new therapeutic strategy using VDZ for CD patients with TB is expected to be established following the accumulation of similar cases.

\section{Abbreviations}

TB: tuberculosis, anti-TNF: anti-tumor necrosis factor, CT: computed tomography, IGRA: interferon gamma release assay, IFX-BS: infliximab-biosimilar, VDZ: vedolizumab, IBD: inflammatory bowel disease, WBC: white blood cell, CRP: C-reactive protein,

SBCE: small bowel capsule endoscopy, PCR: polymerase chain reaction, CDAl: Crohn's disease activity index, SES-CD: simple endoscopic score for Crohn's disease, AOCC: Asian Organization for Crohn's and Colitis, TST: tuberculin skin test, MAdCAM-1: mucosal addressin cell adhesion molecule-1

\section{Declarations}

\section{Ethics approval and consent to participate}

Not applicable.

Consent for publication

Written informed consent was obtained from the patient for publication of this case report and accompanying images. A copy of the written consent is available for review by the Editor-in-Chief of this journal. 
All data generated or analyzed during this study are included in this published article.

\section{Competing Interests}

Y. Sugiyama, S. Tachibana, N. Ueno, Y. Kobayashi, Y. Murakami, T. Sasaki, T. Kunogi, K. Takah

ashi, K. Ando, S. Kashima, K. Moriichi and H. Tanabe declare no conflicts of interest in association with the present study. T. Okumura has received grants from AbbVie Inc, Astellas Pharma Inc., SHIONOGI Co. Ltd., EISAI Co. Ltd. and Hokkaido Welfare Federation of Agricultural Cooperatives. M. Fujiya received grants from JIMRO Co. Ltd., and Kamui Pharma Inc.; grants and personal fees from AbbVie Inc, AYUMI Pharmaceutical Corporation, EA Pharma Co. Ltd., Janssen Pharmaceutical K.K., Kyorin Pharmaceutical Co. Ltd., Mitsubishi Tanabe Pharma Corporation, Mochida Pharmaceutical Co. Ltd., Nippon Kayaku Co. Ltd., Pfizer Inc, Takeda Pharmaceutical Co. Ltd., and ZERIA Pharmaceutical Co. Ltd.; and personal fees from Aspen Japan K.K., Kissei Pharmaceutical Co. Ltd., and Olympus Co. Ltd.

\section{Funding}

This case report has no funding sources.

\section{Authors' contributions}

NU designed entire treatment. YS, ST, YK, YM and KA were in charge of the management of patient's hospitalization. TK, TS, KT, SK and HT performed the endoscopic procedure and cross-section imaging. $\mathrm{KM}, \mathrm{NU}$ and MF were in charge of the pathological diagnosis. All authors discussed the patient's clinical diagnosis and treatment plan. YS and NU wrote the manuscript. TO and MF reviewed the manuscript. All authors approved the final version of this manuscript.

\section{Acknowledgements}

Not applicable.

\section{References}

1. Bonovas S, Fiorino G, Allocca M, Lytras T, Nikolopoulos GK, Peyrin-Biroulet L, et al. Biologic Therapies and Risk of Infection and Malignancy in Patients With Inflammatory Bowel Disease: A Systematic Review and Network Meta-analysis. Clin Gastroenterol Hepatol. Elsevier, Inc; 2016;14:1385-97.e10.

2. Souto A, Maneiro JR, Salgado E, Carmona L, Gomez-Reino JJ. Risk of tuberculosis in patients with chronic immune-mediated inflammatory diseases treated with biologics and tofacitinib: A systematic review and meta-analysis of randomized controlled trials and long-term extension studies. Rheumatol (United Kingdom). 2014;53:1872-85. 
3. Keane J, Gershon S, Wise RP, Mirabile-Levens E, Kasznica J, Schwieterman WD, Siegel JN, Braun MM. Tuberculosis associated with infliximab, a tumor necrosis factor alpha-neutralizing agent. N Engl J Med. 2001 Oct 11;345(15):1098 - 104.

4. Lamb CA, Kennedy NA, Raine T, Hendy PA, Smith PJ, Limdi JK, et al. British Society of Gastroenterology consensus guidelines on the management of inflammatory bowel disease in adults. Gut. 2019;68:s1-106.

5. Ran Z, Wu K, Matsuoka K, Jeen YT, Wei SC, Ahuja V, et al. Asian Organization for Crohn's and Colitis and Asia Pacific Association of Gastroenterology practice recommendations for medical management and monitoring of inflammatory bowel disease in Asia. J Gastroenterol Hepatol. 2020;1-9.

6. Park D, Hisamatsu T, Chen M, Ng SC, Ooi CJ, Wei SC, et al. Asian organization for Crohn's and Colitis and Asia Pacific Association of Gastroenterology consensus on tuberculosis infection in patients with inflammatory bowel disease receiving anti-tumor necrosis factor treatment. Part 1: Risk assessment. Intest Res. 2018;16:4-16.

7. Lamb CA, Kennedy NA, Raine T, Hendy PA, Smith PJ, Limdi JK, et al. British Society of Gastroenterology consensus guidelines on the management of inflammatory bowel disease in adults. Gut. 2019;68:s1-106.

8. Colombel JF, Sands BE, Rutgeerts P, Sandborn W, Danese S, D'Haens G, et al. The safety of vedolizumab for ulcerative colitis and Crohn's disease. Gut. 2016;66:839-51.

9. Lu P, Chen X, Zhu L, mei, Yang H tao. Interferon-Gamma Release Assays for the Diagnosis of Tuberculosis: A Systematic Review and Meta-analysis. Lung. Springer US; 2016;194:447-58.

10. Santos JA, Duarte R, Nunes C. Host factors associated to false negative and indeterminate results in an interferon- $y$ release assay in patients with active tuberculosis. Pulmonology Sociedade Portuguesa de Pneumologia. 2020;26:353-62.

11. Shah SC, Colombel JF, Sands BE, Narula N. Systematic review with meta-analysis: Mucosal healing is associated with improved long-term outcomes in Crohn's disease. Aliment Pharmacol Ther. 2016;43:317-33.

12. Danese S, Sandborn WJ, Colombel JF, Vermeire S, Glover SC, Rimola J, et al. Endoscopic, Radiologic, and Histologic Healing With Vedolizumab in Patients With Active Crohn's Disease. 157: Gastroenterology. Elsevier, Inc; 2019. 1007-18.e7.

13. Cohen RD, Bhayat F, Blake A, Travis S. The Safety Profile of Vedolizumab in Ulcerative Colitis and Crohn's Disease: 4 Years of Global Post-marketing Data. J Crohn's Colitis. 2020;14:192-204.

14. Ramos GP, Stroh G, Al-Bawardy B, Faubion WA, Papadakis KA, Escalante P. Outcomes of treatment for latent tuberculosis infection in patients with inflammatory bowel disease receiving biologic therapy. Inflamm Bowel Dis. 2018;24:2272-7.

\section{Figures}



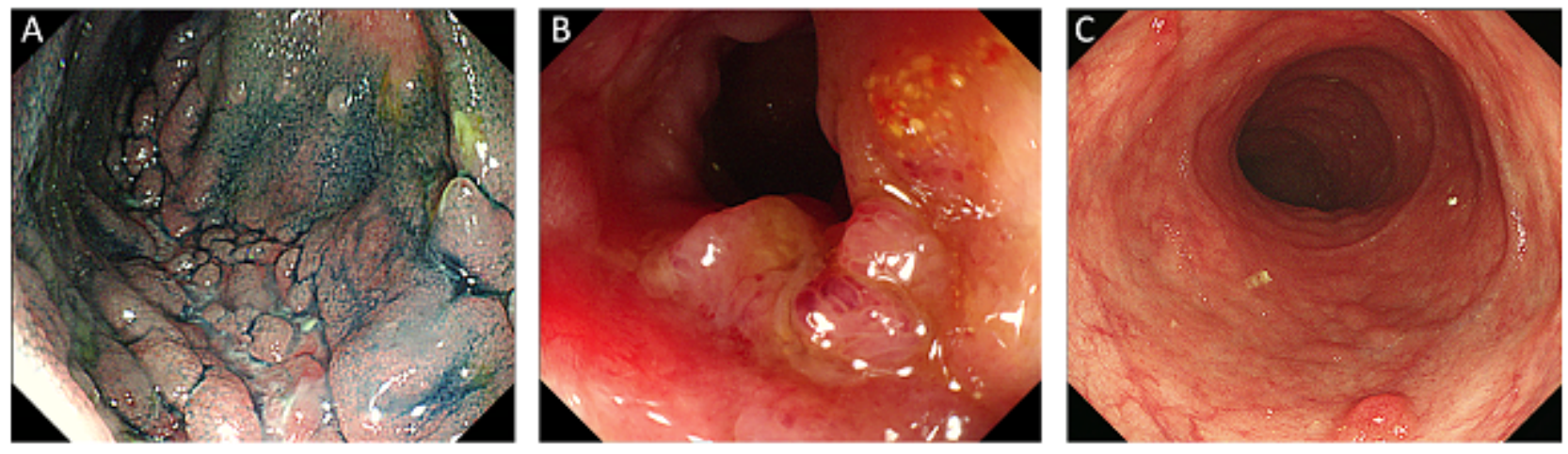

\section{Figure 1}

Endoscopic findings. A: Multiple longitudinal ulcers in the terminal ileum at the initial diagnosis. B: Persistent active ulcer in the terminal ileum after the administration of anti-tuberculosis therapy. C: Mucosal healing in the terminal ileum one year after the administration of VDZ.

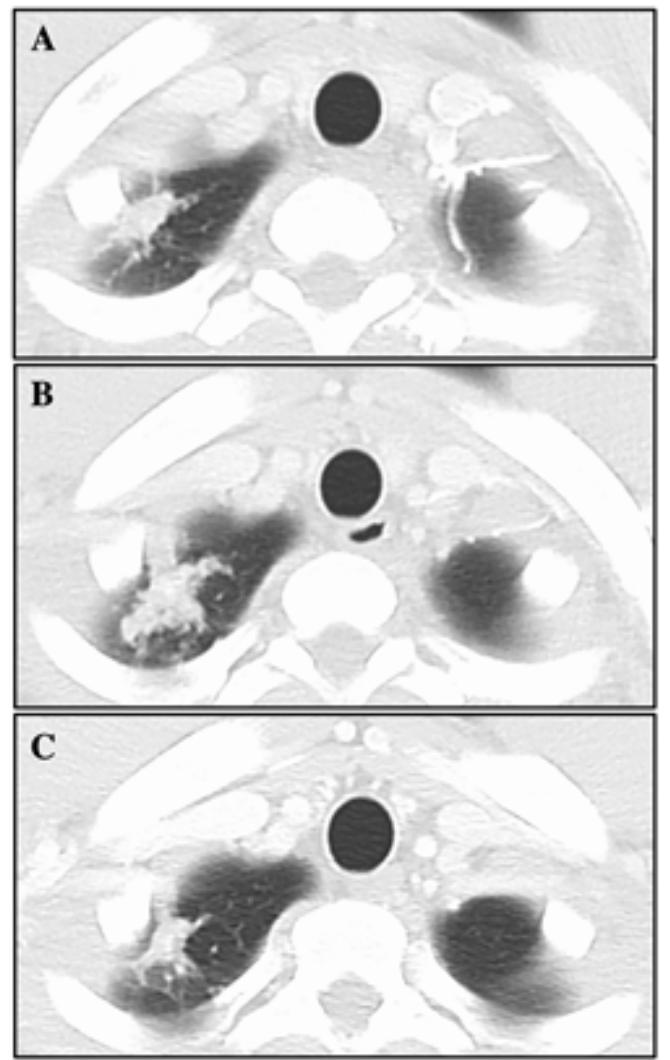

Figure 2

Chest computed tomography findings. A: A nodular lesion at the apex of the right lung before the administration of IFX-BS. B: A worsening nodular lesion 17 weeks after the initiation of IFX-BS. C: A shrunken nodular lesion one year after the administration of VDZ. 